\title{
Author Identification in Bengali Literary Works
}

\author{
Suprabhat Das and Pabitra Mitra \\ Department of Computer Science and Engineering \\ Indian Institute of Technology Kharagpur \\ West Bengal, Pin - 721302, India \\ \{suprabhat, pabitra\}@cse.iitkgp.ernet.in
}

\begin{abstract}
In this paper, we study the problem of authorship identification in Bengali literary works. We considered three authors namely Rabindranath Tagore, Bankim Chandra Chattopadhyay and Sukanta Bhattacharyay. It was observed that simple unigram and bi-gram features along with vocabulary richness were rich enough to discriminate amongst these authors. Although results degraded slightly when training set size was considerably small. For larger training set, a classification accuracy of above $90 \%$ for unigram feature and almost $100 \%$ for bi-gram feature was achieved. Results could be improved further by using more sophisticated features.
\end{abstract}

Keywords: Stylometry, authorship attribution, Bengali literary works, unigram, bi-gram.

\section{Introduction}

Stylometry is the study of the unique linguistic styles and writing behaviors of individuals. Author identification is one of the important problems in stylometrics and it can be seen as a single-label multi-class text categorization problem. It has many academic and literary applications, like author verification, plagiarism detection, genre classification etc. It has legal applications too, like forensic linguistics, detection of genuine confessions. In the last few years it has successfully been applied to broader areas, ranging from blogs, forums, wikis, email, chat and other forms of digital content to music and fine-art paintings.

Stylometry has been studied on English for long time. It was started by Mendenhall [1], in the 19th century, with his work on the plays of Shakespeare. He had reported few authorship attribution method by some characteristic curves, based on sentence length counts and word length counts. It was followed by some statistical studies by Zipf [2] and Yule [3] in the first half of 20th century. One of the most influential works in authorship attribution was done by Mosteller and Wallace [4] on the authorship of 'The Federalist Papers' (a series of articles published in 1787-88, written by John Jay, Alexander Hamilton and James Madison). They had employed Bayesian statistical analysis on a small set of function words such as prepositions, conjunctions and articles as discriminators. Burrows 56] first applied multivariate analysis (MVA) and principle 
components analysis (PCA) on some function words for attributing authorship, followed by Binongo \& Smith [7, Holmes et al. 8] and also by Burrows [910] to resolve many authorship problems. Kjell et al. 11 used neural networks and k-nearest neighbors on character n-grams, whereas Baayen et al. [12] used only neural networks on the syntax of the sentence. Juola \& Baayen [13] used cross entropy as classification method on function words. Zhao \& Zobel 14 reported a different distance measure on function words and part-of-speech (POS) tags. Stamatatos [15] used support vector machines (SVM) on character n-grams to classify English and Arabic news corpus. Koppel et al. [16] reported approach of authorship attribution for thousands of candidate authors.

In Bengali no major work has been done. Mansur et al. 17. proposed an n-gram based text categorization algorithm and also analyzed its efficiency on few Bengali newspaper corpus. We make initial attempts on a collection of documents consisting of Rabindranath Tagore, Bankim Chandra Chattopadhyay and Sukanta Bhattacharyay. We found that using unigram and bi-gram words and vocabulary richness we could achieve a satisfactory result.

The rest of this paper is organized as follows: Section 2 gives a brief overview of stylometric features we have used for evaluation. In Section 3, we focus on the classifier algorithm. The details of the collection are given in Section 4. Section [5] describes the details of the experimental results. In Section 6, we have concluded about our evaluation result and also about some other features that can be included in future works for the betterment of our research work.

\section{Stylometric Features}

A wide variety of relevant features have been reported in many earlier works. Stamatatos 18 has surveyed on almost all stylometric features used till now and modern authorship attribution methods. There are different types of stylometric features to quantify the writing style. Character features are the simplest and basic stylometric feature, as this can be applied in any natural language without any prior knowledge about the language. Lexical features are most commonly used feature for authorship attribution problems whereas syntactic and semantic features are used for more advanced and complicated tasks like POS tagging, parsing. Application-specific features are used for some specialized applications, like lemmatizer or specialized dictionaries.

Feature selection and extraction is one of the biggest challenges in authorship attribution problems. Many types of features are often combined to select respective feature set. Some lexical features are used to build the feature set in our experiment. In first stage of the experiment, unigram words and vocabulary richness are taken as the feature set to quantify the authorship. A relevant tokenizer is used to segment text into tokens or unigram words. Punctuation marks, white space, mathematical notations and special characters are taken as separator to the consecutive tokens. Bi-gram words are nothing but combination of two consecutive unigram words. Bi-gram words and vocabulary richness are combined to use as feature set in the next stage of the experiment. For example, 
the test sentences "John is a good boy. He likes to play cricket" would be composed of following unigram and bi-gram words, tabulated in Table 1 .

Table 1. Unigram and bi-gram words from the test sentence

Unigrams John, is, a, good, boy, He, likes, to, play, cricket \begin{tabular}{|l|l|}
\hline Bi-grams & John is, is a, a good, good boy, boy He, He likes, likes to, to play, play cricket \\
\hline
\end{tabular}

Vocabulary richness is a measurement of diversity of the vocabulary of a text. It is defined by $\frac{V}{N}$, where $V$ is number of unique tokens and $N$ is the total number of tokens of the text. An example of calculating vocabulary richness from the test sentences "Tom always harasses Jerry. Jerry is intelligent. Mickey is friend of Jerry" is given in the Table 2 .

Table 2. Frequencies of unigram words and corresponding vocabulary richness

\begin{tabular}{|l|c|c|}
\hline Unigram words & Frequencies & Vocabulary richness \\
\hline Tom & 1 & $\frac{1}{12}$ \\
\hline always & 1 & $\frac{1}{12}$ \\
\hline harasses & 1 & $\frac{1}{12}$ \\
\hline Jerry & 3 & $\frac{1}{4}$ \\
\hline is & 2 & $\frac{1}{6}$ \\
\hline intelligent & 1 & $\frac{1}{12}$ \\
\hline Mickey & 1 & $\frac{1}{12}$ \\
\hline friend & 1 & $\frac{1}{12}$ \\
\hline of & 1 & $\frac{1}{12}$ \\
\hline
\end{tabular}

\section{Classifier Algorithm}

Classification is the next important step after feature selection. Test documents are attributed to a known author from the set of candidate authors. Starting from very basic statistical measures, like distance measure, Naive Bayes classifier, some advanced measures like neural networks, k-nearest neighbors are also used to quantify authorship. In our experiment, we have used a probabilistic classification method, which is variation of Naive Bayes classification method [19]. In our experiment, classification feature is measured by a simple probability metric

$$
P_{A}=\frac{S_{A}}{F_{A} \times T}
$$

where, $P_{A}$ is probability of test document being written by Author $A, S_{A}$ is total occurrence of test document tokens in training set of Author $A, F_{A}$ is total frequency of tokens in training set of the corresponding author and $T$ is total number of tokens in test document.

In the above formula, $T$, being a constant denominator, will have no effect on the probability $P_{A}$ in a relative scale. The division by $T$ will normalize the 
probability for documents of different size and it will be effective only when we would like to find out the more likely document for a candidate author from a set of test documents.

To calculate the probability metric of a test document for a candidate author, we need to find out correct tokens from test and training document first. In our experiment, initially unigram words are considered as the feature set for classification. In this step, all unigram words are taken as individual tokens to calculate the simple probability metric $P_{A}$ mentioned earlier. In the next step, bi-gram words are considered as the feature set for classification and all the bi-gram words are taken as individual tokens to calculate the same.

The metric measures the probability of a test document being written by a candidate author. This classification metrics are calculated for all candidate authors to quantify the authorship of a test document. A test document is attributed to a candidate author, if the classification metric for that author is greater than that of other candidate authors.

Suppose there are $\mathrm{N}$ candidate authors $\left(A_{1}, A_{2}, \ldots, A_{N}\right)$ for a test corpus. $\mathrm{A}$ test document is attributed to a candidate author by using the following formula.

$$
\text { If }\left(P_{A i}>P_{A j}\right) \text { for } j=1 \text { to } N \text { and } i \neq j
$$

Test document is attributed to author $A_{i}$

The authorship attribution approaches are distinguished in mainly two ways according to the extraction of authors' style cumulatively or individually. In profile-based approach, all the training texts per author are concatenated in a file. On the other hand, each training text is individually represented in separate files for instance-based approach. We prefer instance-based approach for attributing authorship from the test collection.

\section{Details of the Collection}

We used test collection of Bengali documents consisting of Rabindranath Tagore and Bankim Chandra Chattopadhyay's novels and Sukanta Bhattacharyay's poems in our experiment. There are total 36 documents (13 novels by Rabindranath Tagore (RT), 14 novels by Bankim Chandra Chattopadhyay (BCC) and 9 poems by Sukanta Bhattacharyay (SB)) in the complete set of test collection. Detailed statistics of the test collection are given in the Table 3 .

Table 3. Detailed statistic of the test collection

\begin{tabular}{|l|c|c|c|c|}
\hline Author Name & Genre & No. of Docs & Total No. of Tokens & No. of Unique Tokens \\
\hline Rabindranath Tagore (RT) & Novel & 13 & 594757 & 40626 \\
\hline Bankim Chandra Chattopadhyay (BCC) & Novel & 14 & 381119 & 36016 \\
\hline Sukanta Bhattacharyay (SB) & Poem & 9 & 1093 & 691 \\
\hline
\end{tabular}

There are several steps in our experiment. In each steps, we choose $30 \%, 50 \%$ and $70 \%$ documents respectively from the collection of each candidate authors 
as training data set. The selection of training data set was done randomly. The remaining documents are attributed on the basis of this training data set. Division of the whole corpus into training set and test set are tabulated in Table 4 . when $30 \%, 50 \%$ and $70 \%$ of data from individual authors are used for training.

Table 4. Division of whole corpus into training set and test set when $30 \%, 50 \%$ and $70 \%$ data used for training

\begin{tabular}{|l|l|l|l|l|l|l|l|l|}
\hline \multirow{2}{*}{$\begin{array}{l}\text { Author } \\
\text { Name }\end{array}$} & $\begin{array}{l}\text { No. of } \\
\text { docs }\end{array}$ & $\begin{array}{l}\text { No\% data used for training } \\
\text { training set }\end{array}$ & $\begin{array}{l}\text { No. of docs data used for training } \\
\text { in test set }\end{array}$ & $\begin{array}{l}\text { No. of docs in data used for training } \\
\text { training set }\end{array}$ & $\begin{array}{l}\text { No. of docs } \\
\text { in test set }\end{array}$ & $\begin{array}{l}\text { No. of docs in } \\
\text { training set }\end{array}$ & $\begin{array}{l}\text { No. of docs } \\
\text { in test set }\end{array}$ \\
\hline RT & 13 & 4 & 9 & 7 & 6 & 9 & 4 \\
\hline BCC & 14 & 4 & 10 & 7 & 7 & 10 & 4 \\
\hline SB & 9 & 3 & 6 & 4 & 18 & 6 & 3 \\
\hline Total & 36 & 11 & 25 & 18 & 18 & 25 & 11 \\
\hline
\end{tabular}

\section{Experimental Results}

All the experiments have been done on the test corpus, mentioned earlier. In the first stage of our experiment, unigram words and vocabulary richness are taken as feature set. In this case at first $30 \%$ of documents from the collection of each candidate authors are selected randomly to train the classifier. So out of total 36 documents, 11 documents are selected for training set. Remaining 25 documents are classified on the basis of the training data set. In the next step, $50 \%$ and $70 \%$ data from each author are used for training and remaining documents are classified. Lastly each and every document is classified when all the remaining documents are used as training set. The percentages of correctly classified documents, taken unigram words as the feature set, are tabulated in the Table 5 .

Table 5. Rate of correctly classified documents when unigram words are taken as feature set

\begin{tabular}{|l|l|l|l|}
\hline No. of docs in training set & $\begin{array}{l}\text { No. of docs to } \\
\text { be classified }\end{array}$ & $\begin{array}{l}\text { No. of correctly } \\
\text { classified docs }\end{array}$ & $\begin{array}{l}\text { Percentage of cor- } \\
\text { rect classification }\end{array}$ \\
\hline $11(30 \%$ from each candidate authors $)$ & 25 & 19 & $76.00 \%$ \\
\hline $18(50 \%$ from each candidate authors $)$ & 18 & 14 & $77.78 \%$ \\
\hline $25(70 \%$ from each candidate authors $)$ & 11 & 9 & $81.82 \%$ \\
\hline 35 (All the documents except the test case) & 36 & 33 & $91.67 \%$ \\
\hline
\end{tabular}

From this observation, it is seen that the percentage of correct classification is improved with increasing size of training set.

In the next stage, same thing has been done except the selection of feature set. As feature set, bi-gram words and vocabulary richness are selected in this case. Similarly $30 \%, 50 \%$ and $70 \%$ data from individual authors is trained to classify remaining documents on the basis of new feature set. Then every document is 
classified separately when remaining documents are used as training data. In this case, there was a huge improvement in the percentage result. The percentages of correctly classified documents, taken bi-gram words as the feature set, are tabulated in the Table 6.

Table 6. Rate of correctly classified documents when bi-gram words are taken as feature set

\begin{tabular}{|l|l|l|l|}
\hline No. of docs in training set & $\begin{array}{l}\text { No. of docs to } \\
\text { be classified }\end{array}$ & $\begin{array}{l}\text { No. of correctly } \\
\text { classified docs }\end{array}$ & $\begin{array}{l}\text { Percentage of cor- } \\
\text { rect classification }\end{array}$ \\
\hline $11(30 \%$ from each candidate authors $)$ & 25 & 25 & $100.00 \%$ \\
\hline $18(50 \%$ from each candidate authors $)$ & 18 & 18 & $100.00 \%$ \\
\hline $25(70 \%$ from each candidate authors $)$ & 11 & 11 & $100.00 \%$ \\
\hline 35 (All the documents except the test case $)$ & 36 & 36 & $100.00 \%$ \\
\hline
\end{tabular}

From this observation, it is obvious that as a feature set, bi-gram words are good enough to classify authorship of the Bengali corpus. Even a small size of training data can quantify the authorship of test documents correctly.

\section{Conclusion}

It is evident from our experiment that the attribution results strongly depend on the size of test corpus as well as proper feature set selection. In our experiments, only some lexical features are taken into account. Using some advanced feature or combination of some features on Bengali test collection may result better. Many machine learning techniques like principle components analysis (PCA), support vector machines (SVM) can be used to quantify authorship of Bengali test documents in recent future. The application of the well-known tf-idf (term frequency*inverse document frequency) 20 principle for unigram and bi-gram features can also be useful. The main problem with the resource is the unavailability of Bengali literary works by different authors. The three candidate authors in our experiment (RT(1861-1941), BCC(1838-1894) and SB(1926-1947)) belong to distinct generation. So there are some differences in their vocabulary and style. The availability of many documents by different candidate authors from the same age and of similar style will validate the effectiveness of this method. In general, this result can be generalized to classify blog writers, detect plagiarism in Bengali also.

\section{References}

1. Mendenhall, T.C.: The characteristic curves of composition. Science ns-9, 237-246 (1887)

2. Zipf, G.K.: Selected Studies of the Principle of Relative Frequency in Language. Harvard University Press, Cambridge (1932)

3. Yule, G.U.: The Statistical Study of Literary Vocabulary. Cambridge University Press, Cambridge (1944) 
4. Mosteller, F., Wallace, D.L.: Inference and Disputed Authorship: The Federalist. Addison-Wesley, Reading (1964)

5. Burrows, J.F.: Word patterns and story shapes: The statistical analysis of narrative style. Literary and Linguistic Computing 2, 61-70 (1987)

6. Burrows, J.F.: Not unles you ask nicely: The interpretative nexus between analysis and information. Literary and Linguistic Computing 7, 91-109 (1992)

7. Binongo, J.N.G., Smith, M.W.A.: The application of principal component analysis to stylometry. Literary and Linguistic Computing 14, 445-466 (1999)

8. Holmes, D.I., Robertson, M., Paez, R.: Stephen crane and the new-york tribune: A case study in traditional and non-traditional authorship attribution. Computers and the Humanities 35, 315-331 (2001)

9. Burrows, J.F.: Delta: a measure of stylistic difference and a guide to likely authorship. Literary and Linguistic Computing 17, 267-287 (2002)

10. Burrows, J.F.: The englishing of juvenal: Computational stylistics and translated texts. Style 36, 677-699 (2002)

11. Kjell, B., Woods, W.A., Frieder, O.: Information retrieval using letter tuples with neural network and nearest neighbor classifiers. In: IEEE International Conference on Systems, Man and Cybernetics, Vancouver, BC, vol. 2, pp. 1222-1225 (1995)

12. Baayen, H., Van Halteren, H., Tweedie, F.: Outside the cave of shadows: Using syntactic annotation to enhance authorship attribution. Literary and Linguistic Computing 11, 121-132 (1996)

13. Juola, P., Baayen, H.: A controlled-corpus experiment in authorship identification by cross-entropy. Literary and Linguistic Computing 20, 59-67 (2005)

14. Zhao, Y., Zobel, J.: Searching with style: Authorship attribution in classic literature. In: Proceedings of 30th Australasian Conference on Computer Science, vol. 62, pp. 59-68 (2007)

15. Stamatatos, E.: Author identification: Using text sampling to handle the class imbalance problem. Information Processing \& Management 44, 790-799 (2008)

16. Koppel, M., Schler, J., Argamon, S., Messeri, E.: Authorship attribution with thousands of candidate authors. In: Proceedings of the 29th ACM SIGIR, pp. 659-660. ACM Press, New York (2006)

17. Mansur, M., UzZaman, N., Khan, M.: Analysis of n-gram based text categorization for bangla in a newspaper corpus. In: Proceedings of 9th International Conference on Computer and Information Technology, Dhaka, Bangladesh (2006)

18. Stamatatos, E.: A survey of modern authorship attribution methods. Journal of the American Society for Information Science and Technology 60, 538-556 (2009)

19. Mitchell, T.M.: Machine Learning. McGraw-Hill, New York (1997)

20. Manning, C., Raghavan, P., Schutze, H.: Introduction to Information Retrieval. Cambridge University Press, Cambridge (2008) 